\title{
PERFIL DOS MEDICAMENTOS PSICOATIVOS DISPENSADOS NA FARMÁCIA BÁSICA MUNICIPAL DE JI-PARANÁ, RONDÔNIA
}

Profile of psychoative drugs dispensed in Ji-Paraná's municipal Basic Pharmacy, Rondônia

Perfil de los psicofármacos distribuidos en la Farmacia Comunitaria de Ji-Parana, Rondônia

Priscilla Agostinho dos Santos ${ }^{1}$, Tiago Barcelos Valiatti ${ }^{2}$, Jeferson de Oliveira Salvi $i^{1,3^{*}}$

${ }^{1}$ Centro Universitário Luterano de Ji-Paraná (CEULJI/ULBRA

2 Universidade Federal de São Paulo (UNIFESP)

${ }^{3}$ Universidade Luterana do Brasil (ULBRA)

*E-mail: jefersonsalvi@hotmail.com

\section{RESUMO}

Introdução: Psicofármacos modificam seletivamente o Sistema Nervoso Central promovendo alterações no comportamento e no humor.

Objetivo: O presente estudo objetivou caracterizar o perfil dos principais psicoativos dispensados na farmácia básica do município de Ji-Paraná, Rondônia.

Metódos: Realizou-se um estudo transversal por meio do levantamento de dados junto ao sistema Hórus, considerando a análise do período compreendido entre 2013 e 2015.

Resultados: O princípio ativo mais dispensado foi a carbamazepina, seguida pela amitriptilina, fluoxetina, clonazepam e biperideno. A fluoxetina obteve o maior aumento proporcional, seguida pelo clonazepam e pela amitriptilina, tais elevações foram significativas e estiveram associadas com o aumento do número de usuários $(p<0,001)$. A análise comparativa das médias da totalidade dos psicofármacos disponíveis na farmácia básica indicou que apenas entre os anos de 2014 e 2015 não houve um aumento significativo ( $p>0,05)$.

Conclusão: Embora não haja confirmação do uso concomitante por algum dos usuários, todos os fármacos relacionados apresentam potencial para interações medicamentosas entre si, além de requererem cuidados especiais com outras substâncias. A importância do profissional farmacêutico está pautada na gestão dos cuidados durante a dispensação por meio da prestação de esclarecimentos técnicos para os usuários, da observação dos efeitos adversos e interações medicamentosas, contribuindo com uma farmacoterapia racional.

Palavras-chave: Psicofármacos. Farmacovigilância. Farmacoepidemiologia. Uso racional de medicamentos.

\section{ABSTRACT}

Introduction: Psychotropics drugs have the ability to modify selectively the central nervous sytem by promoting changes in the behavior and mood.

Objective: This study aimed to characterize the profile of psychotropic medications dispensed in the basic's pharmacy in the city of Ji-Paraná, state of Rondonia.

Methods: Develops a cross-sectional study through data collection available the Horus system, taking the analysis of the period between 2013 to 2015 .

Results: The most active drug was released carbamazepine, followed by amitriptyline, fluoxetine, clonazepam and biperiden. Fluoxetine represented the greatest proportional increase, followed by clonazepam and the amitriptyline, such elevations represented a significant association with the increase in the number of users $(p<0.001)$. Considering the comparative analysis 
of the average of all psichotropic drugs available in basic pharmacy, just between the years 2014 and 2015 there was no significant ( $p>0.05)$.

Conclusion: All related drugs have potential for interactions between them and requires special care with other substances. The importance of the pharmacist in the management of care during dispensation in pharmacovigilance by the following pharmacotherapeutic and providing technical explanations for users, can be highlighted by the impact of descriptive profile analysis of psychotropic medications available when considering the benefits to health of users with the promotion of the rational use.

Keywords: Psychotropic drugs. Pharmacovigilance. Pharmoepidemiology. Rational use of medications.

\section{RESUMEN}

Introducción: Psicofármacos transforman selectivamente el Sistema Nervioso Central, originando alteraciones en el comportamiento y en el humor.

Objetivo: Este estudio tuvo como objetivo caracterizar el perfil de los principales psicoactivos distribuidosen la farmacia comunitaria de Ji-Paraná, provincia de Rondônia.

Métodos: Se realizó un estudio descriptivo, transversal a través de la recolección de datos por el sistema Horus, teniendo en cuenta el análisis del período entre 2013 y 2015.

Resultado: El fármaco más distribuido fue elcarbamazepina, seguido por la amitriptilina, fluoxetina, clonazepam y biperideno. La fluoxetinarepresentó el mayor aumento proporcional, seguidapor clonazepam y amitriptilina, esaselevaciones fueron significativas y estuvieron asociadas al aumento del número de usuarios $(p<0,001)$. El análisis comparativo de la totalidad de los psicofármacos disponibles en la farmacia básica indicó que apenas entre los años 2014 y 2015 no hubo un aumento significativo ( $p>0,05)$.

Conclusión: Aunque no hay confirmación sobre el uso concomitante en algunos usuarios, todos los fármacos relacionados presentan potencial para interacciones medicamentosas entre sí, además de la necesidad de cuidados especiales con otras sustancias. La importancia del profesional farmacéuticoestá basada en la gestión de los cuidados durante la distribución, a través del ofrecimiento de la información técnica a los usuarios, de la observación de los efectos adversos y de las interacciones medicamentosas, contribuyendo para la construcción de una farmacoterapia racional.

Palabras clave: Psicofármacos. Farmacovigilancia. Farmacoepidemiología. Uso racional de medicamentos.

\section{INTRODUÇÃO}

Medicamentos psicotrópicos são aqueles capazes de promover alterações nos processos mentais, tais como os relacionados ao humor e ao comportamento, modificando de maneira seletiva o Sistema Nervoso Central. Segundo a Organização Mundial da Saúde (OMS) eles podem ser classificados como: ansiolíticos, antidepressivos, antipsicóticos ou neurolépticos, estimuladores psicomotores, psicomiméticos, potencializadores da cognição e sedativos ${ }^{(1)}$.

Embora não se tenha algum dado epidemiológico oficial atualizado sobre o uso de psicotrópicos no Brasil, a classe dos benzodiazepínicos já ocupa a quinta posição do uso abusivo entre substâncias ilícitas(2). Tal representatividade explica-se pela classe estar entre as mais prescritas para o tratamento dos transtornos da ansiedade. Souza e colaboradores ${ }^{(3)}$, avaliando o uso irracional em mulheres, encontraram a associação das indicações com problemas relacionados à ansiedade, insônia e como motivo de fuga da realidade. Embora o contexto da sua utilização envolva a complexidade de vários fatores, existe uma 
tendência do aumento das prescrições devido aos efeitos terapêuticos que proporcionam desde um estado de relaxamento muscular a distintos níveis de sedação e hipnose, além disso, são indicados para os transtornos da ansiedade associados aos distúrbios do sono, convulsões, dependência de álcool e outras substâncias'(4,5,6).

No Brasil, a legislação vigente categoriza os medicamentos psicotrópicos como princípios ativos sujeitos ao controle especial; tais substâncias são descritas em listas atualizadas periodicamente e são apresentadas como notificações de receitas para autorização da dispensação: A3 (coloração amarela), B1 e B2 (colorações azul). Para o registro da comercialização é necessário o uso do Sistema Nacional de Gerenciamento de Produtos Controlados (SNGPC), que corresponde à transmissão de informações para a Vigilância Sanitária com o objetivo de fiscalizar eletronicamente dados sobre a entrada e a saída dos produtos, número de lote, concentração e também dados do prescritor ${ }^{(7)}$.

A Farmacovigilância envolve a identificação, avaliação, compreensão e prevenção de efeitos adversos, além de outros problemas que podem envolver medicamentos, sendo necessário identificar os efeitos e os riscos do uso através do estudo junto à farmacoepidemiologia em uma determinada população(8).

De acordo com Mota et al. ${ }^{(6)}$, a comercialização dos medicamentos está ocorrendo de forma simples e fora do controle, colaborando assim para o uso irracional dos medicamentos, dentre eles os psicotrópicos. Desta forma, a farmacovigilância possui atribuição indispensável no que se refere ao acompanhamento desses medicamentos e juntamente com a farmacoepidemiologia podem descrever, explicar e analisar a utilização e os efeitos da terapêutica medicamentosa.

Há a necessidade da realização de estudos em Farmacoepidemiologia para identificar o porquê, como e para que são prescritos os medicamentos psicotrópicos, a fim de criar métodos para acompanhamento terapêutico dando prioridade aos benefícios e aos efeitos causados pelos medicamentos ${ }^{(9)}$.

O farmacêutico tem papel primordial no acompanhamento da farmacoterapia ao possuir competências e habilidades relacionadas à prestação das informações necessárias para o correto seguimento do tratamento. Tal apontamento sustenta-se pelo registro de que usuários da rede primária pública de saúde anseiam por maiores esclarecimentos sobre as substâncias das quais fazem uso. Tal responsabilidade inicia-se com a correta dispensação, capaz de minimizar as reações adversas e eventuais interações medicamentosas ${ }^{(3,5,11)}$.

O presente estudo objetivou caracterizar os medicamentos psicoativos mais dispensados em uma farmácia básica do interior de Rondônia, considerando relatar as suas principais características farmacológicas.

\section{METODOLOGIA}

Realizou-se um estudo transversal descritivo por meio do levantamento de dados na farmácia básica do município de JiParaná, estado de Rondônia, considerando o período de 2013 a 2015.

Segundo dados do Instituto Brasileiro de Geografia e Estatística (IBGE)(10), a população estimada do município para o ano de 2014 era de 129.242 habitantes. A farmácia básica conta com uma equipe de duas farmacêuticas, duas atendentes de farmácia e uma gerente administrativa. A unidade é centralizada para atender a demanda mensal de aproximadamente 1.200 pessoas, evitando assim que os pacientes percorram as demais 18 Unidades Básicas de Saúde existentes no município.

Os dados foram obtidos junto ao Sistema Hórus, em vigor desde 2009, desenvolvido para qualificar a gestão da assistência farmacêutica no Sistema Único de Saúde (SUS), e apresentados na forma de Unidades Físicas Dispensadas (UFD). Nesse sistema são cadastrados inicialmente dados relacionados aos estabelecimentos de saúde responsáveis pela distribuição e dispensação dos medicamentos disponíveis, assim como as características dos usuários atendidos pelo SUS ${ }^{(11)}$.

Informação do Conselho Federal de Medicina sobre a demografia dos profissionais médicos para o Brasil estipulou que para o estado de Rondônia a razão médico por 1.000 habitantes em 2013 foi de 1,19, aumentando para 1,32 em 2015. Em 
2013, o mesmo índice para médicos cadastrados no SUS foi de 0,75 e destes apenas 13 eram especialistas em psiquiatria, número que passou para 20 em $2015^{(12)}$.

Os princípios ativos identificados foram descritos segundo a denominação comum brasileira $(D C B)^{(13)}$ e relacionados aos principais dados farmacológicos por meio da consulta em literatura específica e pelo software on-line Medscape. As prováveis interações medicamentosas entre os mesmos foram descritas pelo Multi-Drug Interaction Cheker e pelo Drug Interaction Report $^{(14,15)}$.

Os resultados foram submetidos a análise estatística descritiva, sendo que os cinco princípios ativos mais dispensados foram comparados entre si por período por meio da análise de variância (ANOVA, one way) seguida do teste de Tukey para comparações múltiplas, com auxílio do programa GraphPad Prism ${ }^{\circledast}$ (versão 6.0), considerando o nível de significância de $5 \%$.

A pesquisa foi desenvolvida após aprovação prévia do Comitê de Ética em Pesquisa do Centro Universitário Luterano de Ji-Paraná (CEP-CEULJI/ULBRA) por meio do parecer 558.116.

\section{RESULTADOS E DISCUSSÃO}

A Figura 1 indica a distribuição dos cinco psicofármacos mais dispensados nos últimos três anos, a partir do período em que o sistema Hórus foi implantado no estabelecimento.

Figura 1. Distribuição da frequência absoluta dos cinco psicofármacos mais dispensados entre 2013 e 2015 na farmácia básica municipal de Ji-Paraná, Rondônia

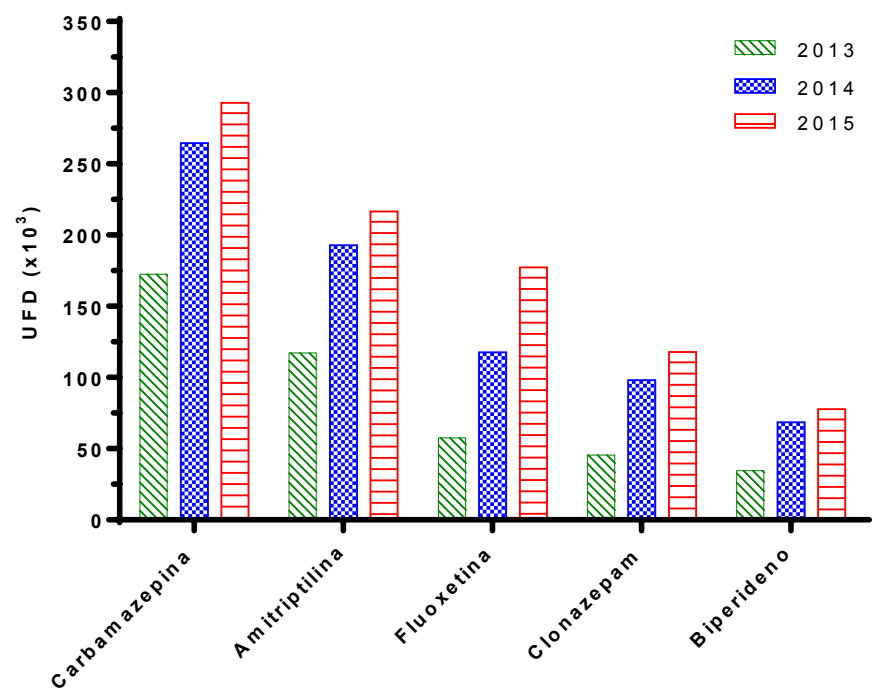

*Apresentações: carbamazepina (comprimido, 200mg), cloridrato de amitriptilina (comprimido, $25 \mathrm{mg}$ ), cloridrato de fluoxetina (cápsula, $20 \mathrm{mg}$ ), clonazepam (comprimido, $2 \mathrm{mg}$ ), cloridrato de biperideno (comprimido, $2 \mathrm{mg}$ ). ${ }^{*}$ teste ANOVA $=p<0,001$, Teste Tukey $=p<0,05$. Fonte: Salvi, 2016. 
Observou-se a existência de uma variação significativa para cada um dos princípios ativos quando comparadas as progressões cronológicas $(p<0,001)$. Considerando, ainda, a comparação múltipla entre os anos, por meio da análise dos valores médios da totalidade dos princípios ativos, apenas entre 2014 e 2015 o aumento de UFD não foi significativo (Teste de Tukey, p>0,05).

A carbamazepina correspondeu ao princípio ativo mais dispensado, porém o aumento da sua dispensação representou aproximados $70 \%$, enquanto que, para a fluoxetina, a frequência relativa para essa mesma proporção foi de $208 \%$, seguida pelo clonazepam (158\%), biperideno (125\%) e pela amitriptilina com $84 \%$. Além disso, se observou a diminuição da tendência do aumento da dispensação do clonazepam em relação aos demais princípios ativos, pois entre 2009 e 2011 ele se manteve em primeiro lugar na maioria do estados, incluindo no de Rondônia ${ }^{(16)}$.

Semelhante aos achados deste estudo, pesquisa realizada em Santa Bárbara (MG)(17) apontou que a Fluoxetina também foi o psicotrópico mais dispensado, seguido pelo clonazempam. Os autores relatam sobre a influência da oferta de diferentes princípios ativos de uma mesma classe na redução do número de unidades físicas dispensadas para fármacos conhecidos pelos prescritores.

Entre 2011 e 2013, em farmácias privadas do mesmo município, estudo de Ravani e Salvi(18) relatou que a amitriptilina e a fluoxetina ocuparam o primeiro e o terceiro lugar, respectivamente, entre os princípios ativos mais dispensados. Ambos os registros corroboram com os resultados para a tendência de aumento segundo os dados farmacoepidemiológicos fornecidos pelo ministério da saúde para o estado de Rondônia ${ }^{(16)}$.

Outra classe identificada foi a dos benzodiazepínicos, unicamente representada pelo clonazepam, para Vale do Paraíso, município da mesma região do estado; esse fármaco ocupou o terceiro lugar entre os psicoativos mais prescritos no ano de $2014^{(19)}$.

Apesar da restrição da utilização do Hórus para a coleta de dados na rede pública, a exigência da informatização dos registros de entrada e saída e da transmissão pelas empresas privadas, propicia a caracterização dos psicofármacos, agregando aos dados farmacoepidemiológicos nacionais.

No Brasil, a Agência Nacional de Vigilância Sanitária demonstra preocupação com o aumento do consumo de fármacos psicoativos e algumas iniciativas sociais, como a denominada medicalização da educação e da sociedade ${ }^{(20)}$, ganham espaço na medida em que esse envolvimento pode influenciar em decisões equilibradas considerando a participação da população e do próprio paciente. $\mathrm{O}$ acesso às informações sobre os psicofármacos pelo público e profissionais de saúde, possibilita a discussão dos cuidados para o uso racional desses medicamentos tal como preconizam as diretrizes de farmacovigilância da União Europeia(21,22).

O aumento da prestação de serviços, considerando o número de UFD para todas as substâncias disponíveis na farmácia, foi associado significativamente ao número de usuários atendidos, estabelecendo uma relação diretamente proporcional $(p<0,0001)$, como demonstra a Tabela 1.

Tabela 1. Relação do número de usuários atendidos com a totalidade dos psicofármacos dispensados em função do período avaliado

\begin{tabular}{cccccc}
\hline ANO & TOTAL DE INDIVÍDUOS ATENDIDOS & MÉDIA & TOTAL DE UFD & MÉDIA & VALOR DE P* \\
\hline $\mathbf{2 0 1 3}$ & 9.509 & 297 & 614,827 & 19.213 & \\
$\mathbf{2 0 1 4}$ & 14.934 & 467 & $1.074,821$ & 33.588 & $<0,0001$ \\
$\mathbf{2 0 1 5}$ & 16.617 & 462 & $1.261,920$ & 35.053 & \\
\hline
\end{tabular}

*Teste do Qui-quadrado de Pearson. UFD = Unidades Físicas Dispensadas.

Fonte: Salvi, 2016.

Tal evidência encontra explicação no próprio crescimento populacional do estado e do município, do número de profissionais prescritores e na melhor efetividade das políticas públicas em fornecer os subsídios para o seguimento farmacoterapêutico específico. Todavia, essas informações sobre o município não estão acessíveis ou completamente disponíveis em um único banco de dados, considerando o histórico do desenvolvimento tecnológico e organizacional da região. 
Na Escócia, um estudo considerou comparar cronologicamente as prescrições dos psicoativos de uma mesma população e concluiu que houve um aumento desproporcional que afetou as classes sociais menos favorecidas, relacionado aos fatores econômicos atribuídos ao custo dos novos princípios ativos que surgiram²(23).

Considera-se também que as enfermidades relacionadas aos transtornos comportamentais vem crescendo em todo o mundo impulsionadas pelos hábitos de vida inerentes aos modelos de desenvolvimento humano. Um estudo realizado na Áustria concluiu que ao longo dos anos as despesas com psicoativos ultrapassaram o investimento destinado as outras áreas da saúde, o que provavelmente esteja associado ao aumento das psicopatologias e das prescrições(24).

A Tabela 2 relaciona os princípios ativos identificados com as principais características farmacológicas, considerando as indicações e as prováveis interações medicamentosas ${ }^{(14,15)}$. Santos et al. ${ }^{(19)}$ evidenciaram que dentre os motivos para o uso de medicamentos controlados, a ansiedade, a depressão e as perturbações do sono, foram as que mais impulsionaram a busca dos pacientes por um diagnóstico e a consequente prescrição de psicoativos.

Tabela 2. Características farmacológicas dos principais psicoativos identificados

\begin{tabular}{|c|c|c|c|}
\hline $\begin{array}{l}\text { PRINCÍPIO ATIVO } \\
\text { (DCB)* }\end{array}$ & $\begin{array}{l}\text { INDICAÇÕES } \\
\text { TERAPÊUTICAS }\end{array}$ & RECOMENDAÇÕES & $\begin{array}{l}\text { INTERAÇÕES } \\
\text { MEDICAMENTOSAS }\end{array}$ \\
\hline Carbamazepina (01710) & $\begin{array}{l}\text { Epilepsia, neuralgia do } \\
\text { trigêmio, síndrome bipolar, } \\
\text { síndrome das pernas } \\
\text { inquietas, esquizofrenia, } \\
\text { neuralgia pós-herpética. }\end{array}$ & $\begin{array}{l}\text { A administração oral deve } \\
\text { ocorrer com alimentos sem que } \\
\text { o comprimido revestido seja } \\
\text { mastigado ou esmagado. }\end{array}$ & $\begin{array}{l}\text { Com fluoxetina: aumento do } \\
\text { efeito da carbamazepina. } \\
\text { Com amitriptilina: diminuição do } \\
\text { efeito da carbamazepina. }\end{array}$ \\
\hline Amitriptilina (00712)\# & $\begin{array}{l}\text { Depressão, neuralgia pós- } \\
\text { herpética, profilaxia da } \\
\text { enxaqueca, desordem } \\
\text { alimentar. }\end{array}$ & $\begin{array}{l}\text { Apresenta fortes efeitos } \\
\text { colinérgicos e pode ocasionar } \\
\text { hipotensão ortostática. }\end{array}$ & $\begin{array}{l}\text { Com clonazepam: aumento do } \\
\text { efeito sedativo. } \\
\text { Com fluoxetina: aumento dos } \\
\text { níveis de seronotina. }\end{array}$ \\
\hline Fluoxetina (04177)\# & $\begin{array}{c}\text { Transtorno depressivo } \\
\text { maior, transtorno obsessivo } \\
\text { compulsivo, bulimia nervosa, } \\
\text { síndrome do pânico, } \\
\text { transtorno disfórico pré- } \\
\text { menstrual, fibromialgia. }\end{array}$ & $\begin{array}{l}\text { O uso não pode ser interrompido } \\
\text { de maneira abrupta devido } \\
\text { a incidência dos sintomas } \\
\text { de abstinência e sintomas } \\
\text { reemergentes. }\end{array}$ & $\begin{array}{l}\text { Com amitriptilina: aumento do } \\
\text { efeito da fluoxetina. } \\
\text { Com carbamazepina: aumento } \\
\text { do efeito da carbamazepina. }\end{array}$ \\
\hline $\begin{array}{l}\text { Clonazepam } \\
(02300) \#\end{array}$ & $\begin{array}{c}\text { Convulsões, hiperplexia, } \\
\text { síndrome do pânico, síndrome } \\
\text { da ardência bucal, transtornos } \\
\text { do sono. }\end{array}$ & $\begin{array}{l}\text { Deve ser evitado no glaucoma } \\
\text { agudo de ângulo fechado, } \\
\text { neurose depressiva, reações } \\
\text { psicóticas, miastenia grave e com } \\
\text { o uso concomitante com álcool. } \\
\text { Deve ser retirado gradualmente. }\end{array}$ & $\begin{array}{l}\text { Com amitriptilina: aumento do } \\
\text { efeito sedativo. } \\
\text { Com carbamazepina: diminuição } \\
\text { do efeito do clonazepam. }\end{array}$ \\
\hline Biperideno (01282)\# & $\begin{array}{l}\text { Mal de Parkinson, distonias } \\
\text { agudas, inquietações, } \\
\text { sintomas extrapiramidais } \\
\text { induzidos por drogas. }\end{array}$ & $\begin{array}{c}\text { Cautela com prostatismo, } \\
\text { epilepsia, arritmias cardíacas e } \\
\text { com o uso concomitante com } \\
\text { álcool. }\end{array}$ & $\begin{array}{l}\text { Com amitriptilina, } \\
\text { carbamazepina e clonazepam: } \\
\text { aumento dos efeitos de } \\
\text { sonolência, boca seca, visão } \\
\text { turva, confusão mental e } \\
\text { problemas de memória. }\end{array}$ \\
\hline
\end{tabular}

*Denominação comum brasileira ${ }^{(21)}$. \#Apresentado na forma de cloridrato.

Fonte: Salvi, 2016 
A carbamazepina é um medicamento anticonvulsivante que bloqueia canais de sódio suprimindo a convulsão ao impedir o desvio polarizante paroxístico. A sua utilização pode estar relacionada à síndrome de Stevens-Johnson, anemia aplásica, agranulocitose e leucopenia ${ }^{(25)}$. Alguns autores sugerem a sua melhor eficácia em relação à fenitoína, embora ambas sejam consideradas seguras, existindo também registros dos seus benefícios como monoterapia ou medicamento adjuvante para o controle da síndrome da abstinência ao álcool, da dor e como estabilizadora do humor ${ }^{(26,27)}$.

A amitriptilina pertence à classe dos antidepressivos tricíclicos, seu mecanismo de ação se dá pelo bloqueio da recaptação da serotonina e da noradrenalina. A sua prescrição é indicada para os estados depressivos em casos de transtornos do humor, os efeitos adversos mais comuns são dor abdominal, sedação, tontura, fadiga e visão embaçada. Portella et al. ${ }^{(28)}$ encontraram resultados positivos no tratamento do Herpes-Zóster, utilizado para o controle e alívio da dor, e na melhora do autocuidado em casos de fibromialgia ${ }^{(29)}$. Alguns estudos ainda apontam para o seu uso inadequado, principalmente entre pacientes idosos, como registraram Narayan e Nishtala ${ }^{(30)}$ na Nova Zelândia. A associação com a fluoxetina é considerada moderada, diferentemente do uso associado com a Risperidona que é classificado como grave ${ }^{(31)}$.

A fluoxetina exerce o seu efeito principal inibindo a recaptação de serotonina, mas estudos indicam a sua interação com outros receptores localizados no cérebro. A sua utilização concomitante com inibidores da MAO (IMAO) pode ser fatal, estabelecendo-se um intervalo de no mínimo quatorze dias após a interrupção desse para o início do tratamento com a fluoxetina. A associação com tioridazina, indicada para tratamento da esquizofrenia crônica, pode induzir a problemas cardíacos por aumentar o intervalo QT. A utilização com Varfarina pode inibir o metabolismo dessa devido às interações com as isoenzimas do citocromo p450(32, 33).

No Brasil, o clonazepam está presente sob a forma de 68 diferentes formulações prescritas por meio de notificação de receita de cor azul. É classificado legalmente como medicamento psicotrópico, pois o seu uso pode estabelecer a dependência física ou psíquica. Seu mecanismo de ação ocorre pela ação agonista sobre os receptores gabaérgicos do tipo ao aumentar o influxo de íons cloro. No ano de 2013 o seu consumo global declarado representou 8,9 bilhões de toneladas. Neste mesmo período o Brasil produziu 3,2 bilhões de toneladas, passando a ser o maior fabricante mundial segundo dados da Organização das Nações Unidas ${ }^{(34)}$.

O uso de medicamentos psicotrópicos também pode aumentar o risco de quedas em pacientes idosos, pois nessa faixa etária os efeitos adversos são ainda maiores, além de ser um dos principais motivos associados à prática da polifarmácia e intoxicações ${ }^{(35,36,37)}$. Matta e colaboradores ${ }^{(38)}$ ressaltam que alguns aspectos das exigências legais também podem dificultar ou interferir na qualidade da dispensação e influenciar no correto seguimento farmacoterapêutico.

Em usuários do Sistema Único de Saúde, na cidade de Ribeirão Preto (SP), pesquisadores relataram que entre os problemas relacionados aos medicamentos, a associação de antidepressivos e benzodiazepínicos foi a mais comum, sendo que, a amitriptilina, o clonazepam e a fluoxetina foram os fármacos mais prescritos ${ }^{(39)}$.

O biperideno é indicado para a redução dos sintomas da doença de Parkinson, prevenção dos efeitos dos neurolépticos e há registros de efeitos positivos em casos de dependência de cocaína e crack. Ele exerce a sua ação terapêutica no sistema dopaminérgico por meio da diminuição da recaptação pré-sináptica da dopamina. Em pacientes com Parkinson identificou-se como principal interação medicamentosa a que ocorre entre os antiparkinsonianos e os anti-hipertensivos, seguida de interações entre os próprios representantes da primeira classe citada ${ }^{(40)}$.

Prescrições inadequadas, doses incorretas e a utilização por período prolongado são evidências comumente registradas em pesquisas da área, principalmente em idosos ${ }^{(41,42)}$. Observações como essas podem constituir não apenas um auxílio para a capacitação dos profissionais que lidam ou trabalham com os psicofármacos, como também, ser consideradas para a tomada das decisões no desenvolvimento dos programas de saúde.

O papel da farmacoepidemiologia como instrumento informativo e analítico reflete as diversas possibilidades que buscam explicar o crescimento do consumo de determinado medicamento ou classe terapêutica. A relevância de se compreender tal 
fenômeno reflete a importância da farmacolovigilância como instrumento fomentador de conhecimento junto aos profissionais que atuam direta ou indiretamente com os medicamentos. Dados atualizados direcionam cuidados especiais junto ao ato da dispensação e, consequentemente, colaboram na promoção ao uso racional dos piscofármacos e na melhor adesão à farmacoterapia ${ }^{(43,44)}$.

O breve relato das indicações para os principais psicofármacos dispensados, considerando as classes, mecanismos de ação e prováveis interações, mesmo sem a confirmação do uso concomitante por algum dos indivíduos, abre a possibilidade para a discussão dos possíveis desdobramentos do uso irracional, uma vez que estratégias preventivas apresentam um menor risco à saúde e demonstram-se efetivas para uma maior adesão à farmacoterapia ${ }^{(45,46)}$.

O papel do farmacêutico na orientação sobre os psicofármacos é possível devido à proximidade com o paciente no ato da dispensação ou na prestação dos serviços farmacêuticos, a exemplo da atenção farmacêutica. Embora essa área necessite de mais estudos, resultados satisfatórios foram encontrados sobre a intervenção farmacêutica na atenção à saúde mental ${ }^{(47)}$.

\section{CONCLUSÃO}

A carbamazepina foi o princípio ativo mais dispensado, embora a fluoxetina represente o maior aumento relativo, seguida pelo clonazepam e pela amitriptilina.

Os dados encontrados no presente estudo indicam uma associação significativa diretamente proporcional entre o número de usuários e a quantidade de medicamentos psicofármacos distribuídos em um periódo de tempo relativamente curto. Tal fato pode ter explicação em fatores distintos a serem elucidados em pesquisa futura, considerando caracterizar o perfil dos usuários na busca por compreender a relação de variáveis sociodemográficas com o tempo de uso e o tipo de substância empregada.

A importância do profissional farmacêutico na gestão dos medicamentos psicotrópicos, na farmacovigilância junto ao seguimento farmacoterapêutico e na prestação de esclarecimentos para os usuários no momento da aquisição, é ressaltada pela exigência do controle especial desses princípios ativos que tem como objetivos o uso racional e a promoção da saúde dos pacientes.

\section{REFERÊNCIAS}

1. Firmo WCA, Paredes $A O$, Cunha $C L F$, Torres $A G$, Buccini DF. Análise das prescrições médicas de psicotrópicos de uma farmácia comercial no município de Bacabal, Maranhão. J Manag Prim Health Care, 2013; 4(1):10-18.

2. Telles Filho PCP, Chagas AR, Pinheiro MLP, Lima AMJ, Durao AMS. Utilização de Benzodiazepínicos por idosos de uma Estratégia de Saúde da Família: implicações para a Enfermagem. Esc. Anna Nery 2011; 15(3):581-586.

3. Da Silva TO, Iguti AM. Medicamentos Psicotrópicos Dispensados em Unidade Básica de Saúde em Grande Município do Estado de São Paulo. G\&S, 2013; 4:2004-2015.

4. Brasil. Agência Nacional Da Vigilância Sanitária. Resolução Direcionada Colegiada - RDC No 22, de 29 de abril de 2014 que dispõe Sobre o Sistema Nacional de Gerenciamento de Produtos Controlados - SNGPC. Disponível em: http://www.anvisa. gov.br/sngpc/RDC\%2022\%202014\%20DOU.pdf.

5. Oliveira JR, Xavier RMF, Júnior AFS. Eventos adversos notificados ao Sistema Naciola de Notificações para a Vigilância Sanitária (NOTIVISA):Brasil, estudo descritivo no período 2006 a 2011. Edipemiol. Serv. Saúde, 2013; 22(4):671-678.

6. Mota DM, Costa AA, Teixeira CS, Bastos AA, Dias MF. Uso abusivo de benzidamina no Brasil: uma abordagem em farmacovigilância. Ciên. saúde coletiva, 2010; 15(3):717-724. 
7. Brasil. Ministério da Saúde. Portaria n 344, de 12 de maio de 1998. Regulamento Técnico sobre substâncias e medicamentos sujeitos a controle especial.

8. Asseff IL, Macías MS, Bañuelos REA, Sánchez P, Pérez GG, Castañeda VL, Hernández, CG. Farmacoepidemiología de psicofármacos empleados en la práctica pediátrica en el Servicio de Psiquiatría Infantil del Hospital General de Durango, México. Bol. Med. Hosp. Infant.,2010; 67(1):27-36.

9. Zanella CG, Aguiar PM, Storpirtis S. Atuação do farmacêutico na dispensação de medicamentos em Centros de Atenção Psicossocial Adulto no município de São Paulo, SP, Brasil. Ciênc. saúde coletiva, 2015; 20(2):325-332.

10. IBGE. Instituto Brasileiro de Geografia e estatística. Censo demográfico 2010. Disponível em: http://censo2010.ibge.gov.br/ apps/atlas/

11. Costa KS, Nascimento Jr MJ. HÓRUS: Inovação tecnológica na Assistencia Farmaceutica no Sistema Unico de Saúde. Rev. Saúde Pública, 2012; 46(suppl.1):1-9.

12. Conselho Regional de Medicina do Estado de São Paulo. Demografia Médica no Brasil Vol 2: cenários e indicadores de distribuição. Relatório de pesquisa. 2013. Disponível em: http://www.cremesp.org.br/pdfs/DemografiaMedicaBrasilVol2.pdf

13. Brasil. Agência Nacional de Saúde. Lista DCB 2007. Consolidada. Disponivel em: http://www.anvisa.gov.br/medicamentos/ dcb/lista_dcb_2007.pdf

14. Medscape. Drug Interaction Checker [online]. Disponível em: http://reference.medscape.com/drug-interactionchecker

15. Drug Interaction Checker [online]. Disponível em: http://www.drugs.com/drug_interactions.php

16. Brasil. Agência de Vigilância Sanitária. Boletim Farmacoepidemiológico 2012. Disponível em: http://www.anvisa.gov.br/ hotsite/sngpc/mapainterativo/arquivos/Tabela_RO_6_1_2012.pdf

17. Carvalho EF, Santos JLG. Perfil da dispensação e estratégias para o uso racional de psicotrópicos [Monografia]. Universidade Federal de Santa Catarina, Santa Catarina. Curso de especialização em Linhas de Cuidado em Enfermagem. 2016. Disponível em: https://repositorio.ufsc.br/xmlui/handle/123456789/167287

18. Ravani LC, Salvi JO. Aspectos farmacoepidemiológicos de medicamentos antidepressivos em drogarias do município de Ji-Paraná, Rondônia (Trabalho de Conclusão de curso). Ji - Paraná. Centro Universitário Luterano de Ji - Paraná: Curso de Farmácia. 2014.

19. Santos LP, Oliveira AA, Salvi JO. Farmacovigilância de medicamentos psicotrópicos no município do vale do paraíso, RONDÔNIA. R. Cie. Fac. Educ. E Meio Amb., 2015; 6(2):36-48.

20. Guidelines for good pharmacoepidemiology practices (GPP). [Comentário]. Pharmacoepidemiology and Drug Safety, 2008; 17:200-208.

21. Harayama R, Gomes J, Barros R., Galindo D, Santos D. Nota técnica sobre o consumo de psicofármacos no Brasil. Boletim Farmacoterapêutico, 2015; 19(2):24.

22. Borg JJ, Tanti A, Kouvelas D, Lungu C, Pirozynski M, Serracino-Inglott A, Aislaitner G. European Union pharmacovigilance capabilities: potential for the new legislation. Ther Adv Drug Saf. ${ }_{L} 2015 ;$ 6(4):120-140.

23. Hughes LD, Cochrane L, McMurdo ME, Guthrie B. Psychoactive prescribing for older people-what difference does 15 years make?. Int J Geriatr Psychiatry, 2016; 31(1):49-57.

24. Boeckle M, Chetouani Y, Schrimpf M, Liegl G, Leitner A, Pieh C. Austrian expenditures on psychopharmaceutical drugs between 2006 and 2013. Z Psychosom Med Psychother, 2015; 61(4):359-369. 
25. Golan D, Tashjian Jr AH, Armstrong EJ, Armstrong AW. Princípios de farmacologia: a base fisiopatológica da farmacoterapia. 3. ed. Rio de Janeiro: Guanabara Koogan. 2014.

26. Nolan SJ, Marson AG, Weston J, Tudur Smith C. Carbamazepine versus phenobarbitone monotherapy for epilepsy: an individual participant data review. Cochrane Database Syst Rev. 2 2015; 23(7).

27. Hammond CJ, Niciu MJ, Drew S, Arias AJ. Anticonvulsants for the treatment of alcohol withdrawal syndrome and alcohol use disorders. CNS Drugs, 2015; 29(4):293-311.

28. Portella, AVT, Souza LCB, Gomes JMA. Herpes-zóster e neuralgia pós-herpética. Rev Dor, 2013;14(3):210-215.

29. Kim SC, Landon JE, Lee YC. Patterns of health care utilization related to initiation of amitriptyline, duloxetine, gabapentin, or pregabalin in fibromyalgia. Arthritis Res Ther., 2015; 17(1):1-8.

30. Narayan SW, Nishtala PS. Prevalence of potentially inappropriate medicine use in older New Zealanders: a population-level study using the updated 2012 Beers criteria. J Eval Clin Pract., 2015; 21(4):633-41.

31. Campigotto KF, Teixeira JJV, Cano FG, Sanches ACC, Cano MFF, Guimarães DSL. Deteç̧ão de risco de interações entre fármacos antidepressivos e associados prescritos a pacientes adultos. Rev. psiquiatr. clín., 2008; 35(1):1-5.

32. Food and Drug Administration. Prozac - Fluoxetine Hydrocloride. NDA 18-936/S-064. 2016. 29 p. [online]. Disponível em: http://www.fda.gov/safety/medwatch/safetyinformation/ucm255402.htm

33. Sansone RA, Sansone LA. Warfarin and Antidepressants: Happiness without Hemorrhaging. Psychiatry (Edgmont), 2009; 6(7):24-29.

34. Harayama R, Gomes J, Barros R., Galindo D, Santos D. Nota técnica: o consumo de psicofármacos no brasil, dados do sistema nacional de gerenciamento de produtos controlados anvisa (2007-2014). 2015. Disponível em: <http://medicalizacao.org. br/wp-content/uploads/2015/06/NotaTecnicaForumnet_v2.pdf>

35. Williams LJ, Pasco JA, Stuart AL, Jacka FN, Brennan SL, Dobbins AG. Honkanen R, Koivumaa-Honkanen, H, Rauma, PH, Berk, M. Psychiatric disorders, psychotropic medication use and falls among women: an observational study. BMC Psychiatry 2015;(15):1-7.

36. Baldoni, AO, Ayres, LR, Martinez, EZ, Dewulf, NLS, Santos, V, Obreli-Neto, PR, Pereira, LRL. Pharmacoepidemiological profile and polypharmacy indicators in elderly outpatients. Braz. J. Pharm. Sci., 2013; 49(3): 443-452.

37. Paula, TC, Bochner, R, Montilla, DER. Análise clínica e epidemiológica das internações hospitalares de idosos decorrentes de intoxicações e efeitos adversos de medicamentos, Brasil, de 2004 a 2008. Rev. bras. epidemiol., 2012; 15(4):828-844.

38. Matta SR, Miranda ES, Osorio-de-Castro CGS. Prescrição e dispensação de medicamentos psicoativos nos instrumentos normativos da regulação sanitária brasileira: implicações para o uso racional de medicamentos. Rev. Bras. Farm., 2011; 92(1):33-41.

39. Netto MUQ, Freitas O, Pereira LRL. Antidepressivos e Benzodiazepínicos: estudo sobre o uso racional entre usuários do SUS em Ribeirão Preto-SP. Rev. Ciênc. Farm. Básica Apl., 2012; 33(1):77-81.

40. Lacrimante CA, Almeida DS, Cecconi LC, Crozara MA. Estudo das interações medicamentosas dos antiparkinsonianos no centro de promoção e reabilitação em saúde e integração social - São Camilo. In: Il simpósio de assistência farmacêutica. 2014. São Paulo: Centro Universitário São Camilo. 2014. 
41. Filho JMC, Marcopito LF, Castelo A. Perfil de utilização de medicamentos por idosos em área urbana do Nordeste do Brasil. Rev Saúde Pública, 2004; 38(4):557-564.

42. Firmino KF, Abreu MH, Perini E, Magalhães SM. Factors associated with benzodiazepine prescription by local health services in Coronel Fabriciano, Minas Gerais State, Brazil. Cad Saude Publica, 2011; 27(6):1223-1232.

43. Shirama, FH, Miasso, Al. Consumo de psicofármacos por pacientes de clínicas médica e cirúrgica de um hospital geral. Rev. Latino-Am. Enfermagem, 2013; 21(4): 948-955.

44. Parent G, Rose FX, Bedouch P, Conort O, Charpiat B, Juste M, Roubille R, Allenet B. Pharmacists' interventions conducted by hospital pharmacists on psychotropic drugs pharmacotherapy. Encephale., 2015; 41(4):339-345.

45. Mascarenhas FS, Medeiros-Souza P, Camargos EF, Ferreira F, Silva AR, Homem-de-Mello M. Tablet splitting of psychotropic drugs for patients with dementia: a pharmacoepidemiologic study in a Brazilian sample. Clin Ther., 2015; 37(10):23322338.

46. Rubio-Valera M1, Bosmans J, Fernández A, Peñarrubia-María M, March $M$, Travé $P$, Bellón JA, Serrano-Blanco A. Costeffectiveness of a community pharmacist intervention in patients with depression: a randomized controlled trial (PRODEFAR Study). PLoS One., 2013; 8(8):1-10.

47. Luccheta RC, Mastroianni PC. Intervenções farmacêuticas na atenção à saúde mental: uma revisão. Rev. Ciênc. Farm. Básica Apl., 2012; 33(2):165-169.

Submetido em: 09/08/2016 